\title{
Investigations on direct and maternal genetic components of calving performance in heifers
}

\author{
W. SCHLOTE (1), M. MUNZ (2) and T. HOLZER (2) \\ (1) Institute of Animal Production, Technical University of Berlin, \\ Lentzeallee 75, D-1000 Berlin 33, Germany \\ (2) Institute of Animal Husbandry and Breeding, Hohenheim University, \\ Postfach 700562 (470), D-7000 Stuttgart 70, Germany
}

Data of 1261 heifer daughters sired by 51 testing bulls of a Simmental population were used to estimate population parameters for the direct and maternal components of calving performance and a subset for analyzing interior pelvic measurements. Heritability estimates for the direct and maternal components in the order of $h_{d}^{2}=.10$ to .15 and $h_{m}^{2}=.20$ to .25 , respectively, the correlation estimates floating around zero. Heritabilities for pelvic measurements ranged from $\mathrm{h}^{2}=.29$ to .43 , genetic correlations to calving performance from -.21 to -.35 . Consideration of both components increases accuracy of sire evaluation for calving performance, whereas pelvic measurements provides additional information.

\section{Comparison of genetic parameters for different fertility traits in Simmental cattle}

\author{
J. CHAVAZ and C. GAILLARD \\ Swiss Association for AI, 3052 Zollikofen, Switzerland
}

554.952 first service inseminations were used to estimate the heritability of different fertility traits in Swiss Simmental cattle. The heritability of female fertility traits lies between .9 and $1.7 \mathrm{p} .100$. the best trait being the number of inseminations for a pregnancy. The heritabilities are slightly larger for male fertility traits $(1.1$ to $2.4 \mathrm{p} .100)$; the return after 75 days allows to quantify correctly the differences between bulls. The heritability does not vary much with age of females. some values, however, are slightly higher in cows than in heifers. Comparisons of various fertility traits suggest that differences between bulls come èssentially from the differences in the ability of fertilization, while the manifestation of heats and the embryonic death play also a role in the variability between females.

\section{How to include milkability in dairy cattle breeding}

\author{
F. REINHARDT, L. DEMPFLE and M. SANTOS-CRISTAL \\ Lehrstuhl für Tierzucht der Technischen Universität \\ München, D-8050 Freising-Weihenstephan (Fed. Rep. Ger.)
}

\begin{abstract}
There exists no unique opinion on how to deal with milkability in dairy cattle breeding, nor about its importance. By the present selection method used in Bavaria the positive relationship between milkability and milk yield is not utilized and by adjusting the Milk Flow Rate (MFR) for daily milk yield part of the genetic effects are no longer usuable for selection. Using the results of our own investigations (from 1400 milkability tests) and corresponding values cited in the literature, various selection indices were calculated and discussed. All indices investigated were superior to the index used at present. An index is proposed in which the positive genetic relationship between milk yield and milkability is utilized and in which the MFR is only adjusted for the time interval between calving and test day.
\end{abstract}

